\title{
10 IMPACTO DA CRISE FINANCEIRA E SOCIAL NA SAÚDE MENTAL
}

\author{
| Carlos Sequeira ${ }^{1}$; Luís Sá2; José Carlos Carvalho³ ${ }^{3}$ Francisco Sampaio ${ }^{4}$
}

\section{RESUMO}

Preocupados com o impacto da crise financeira e social na saúde mental das pessoas, neste artigo fazemos uma reflexão sobre as últimas evidências disponíveis sobre o tema em Portugal e no mundo, procurando recentrar a atenção dos profissionais de saúde mental, naquilo que necessita ser promovido: a centralidade dos cuidados nas pessoas e a continuidade dos cuidados em saúde mental. A instalação da crise financeira, que a todos apanhou desprevenidos, veio revelar as fragilidades e falta de preparação dos sistemas de saúde para lidar com problemas sociais que, frequentemente, constituem risco para o surgimento de psicopatologia. A forma como as famílias e as pessoas foram capazes de fazer face às suas necessidades, em termos de bem-estar, é também revelador da capacidade de adaptação e resiliência dos portugueses. A crise financeira e social teve e continuará a ter consequências em várias dimensões da vida das pessoas, pelo que refletimos também sobre algumas estratégias para a minimização dos efeitos da crise na saúde mental das pessoas.

PALAVRAS-CHAVE: Impacto psicossocial; Recessão económica; Saúde mental

\section{RESUMEN}

"Impacto de la Crisis Financiera y Social en la Salud Mental"

Preocupados por el impacto de la crisis financiera y social en la salud mental de las personas, en este artículo se ha reflexionado sobre los últimos datos disponibles sobre el tema, en Portugal y en el extranjero, buscando centrar la atención de los profesionales de salud mental en lo que debe promoverse: la centralidad de la atención en las personas y la continuidad de la atención en salud mental. La instalación de la crisis financiera, que a todos nos pilló con la guardia baja, ha puesto de manifiesto las debilidades y falta de preparación de los sistemas de salud, para hacer frente a los problemas sociales, que a menudo constituyen riesgo para el surgimiento de psicopatología. Las familias y las personas fueron capaces de satisfacer sus necesidades en términos de bienestar, lo que revela también la adaptabilidad y la capacidad de recuperación de los portugueses. La crisis financiera y social ha tenido y seguirá teniendo un impacto en diversas dimensiones de la vida de las personas, por lo que reflexionamos también acerca de algunas estrategias para minimizar los efectos de la crisis en la salud mental.

\section{DESCRIPTORES: Impacto psicosocial; Recesión económica;} Salud mental

\begin{abstract}
"Impact of the Financial and Social Crisis on Mental Health"

We are concerned about the impact of the financial and social crisis in people's mental health. Thus, in this article we decided to reflect on the latest available evidence on the subject in Portugal and abroad, aiming at refocusing the attention of mental health professionals in what we think is necessary to be promoted: the centrality of care in people and the continuity of care in mental health. The financial crisis, which caught everyone off guard, has revealed the weaknesses and lack of preparedness of health systems to deal with social problems that often constitute risk for the emergence of psychopathology. The way families and people were able to meet its needs related to the well-being also reveals the adaptability and resilience of the Portuguese people. The financial and social crisis has had and will continue to have an impact on various dimensions of people's lives, so we also reflected on some strategies for minimizing the effects of the crisis in mental health.
\end{abstract}

\section{KEYWORDS: Psychosocial impact; Economic recession; Men- tal health}

Submetido em 30-09-2015

Aceite em 30-11-2015

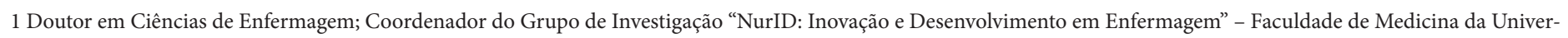

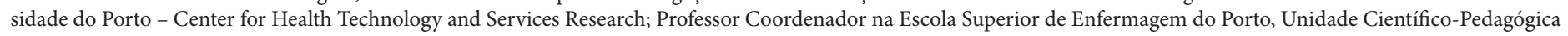
"Gestão de Sinais e Sintomas", Rua Dr. António Bernardino de Almeida, 4200-072 Porto, Portugal, carlossequeira@esenf.pt

2 Doutor em Saúde Mental; Professor Auxiliar na Universidade Católica Portuguesa, Instituto de Ciências da Saúde, 4200-072 Porto, Portugal, lsa@porto.ucp.pt

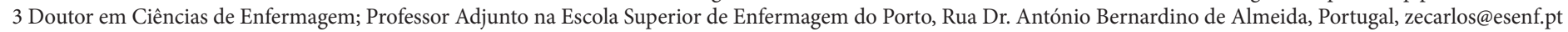

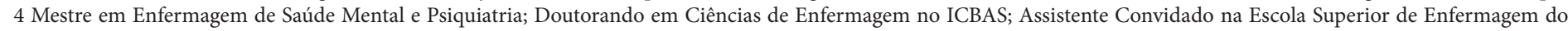

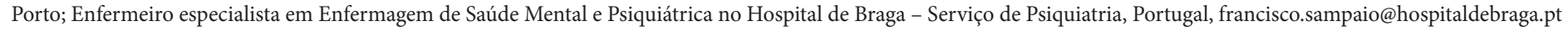




\section{CONTEXTUALIZAÇÃO}

Desde o ano 2008 vive-se, a nível mundial, uma crise financeira que ainda se faz sentir nos dias de hoje, precipitada pela falência do banco de investimento norteamericano Lehman Brothers, fenómeno que já foi internacionalmente denominado "Great Recession”. Em "efeito dominó", outras importantes instituições financeiras tiveram também quebras significativas, num processo também conhecido como "crise dos subprimes". Em Portugal, os efeitos desta crise começaram a ser sentidos de forma mais significativa a partir de 2010, em consequência da maior contração económica da história da Zona Euro (quebra de 1,5\% do Produto Interno Bruto no quarto trimestre de 2008, em relação ao trimestre anterior) e de políticas económicas nacionais voltadas para o aumento da despesa pública e do investimento público.

Como forma de dar resposta à crise financeira, a União Europeia (bem como outras organizações internacionais) decidiu apostar no incentivo à adoção de políticas de austeridade, tendo em vista a recuperação económica dos países mais afetados pela mesma (sobretudo os países do Sul da Europa, onde se inclui Portugal).

Apesar de, em termos financeiros, as medidas implementadas terem surtido algum efeito, os custos das mesmas fizeram-se sentir de forma significativa a nível social, podendo mesmo considerar-se que, apesar do seu ponto de partida financeiro, a crise que afetou (e que ainda se faz sentir) Portugal foi, igualmente, uma crise social.

No plano internacional, muito se têm estudado os efeitos da crise financeira na vida das pessoas, na sua saúde e, particularmente, na sua saúde mental. Assim, sabese por exemplo que as políticas de austeridade vieram agravar as desigualdades socioeconómicas, havendo investigação que sugere, por exemplo, a existência de diferenças na estrutura cerebral das crianças que vivem em situação de pobreza, relativamente às outras crianças, sendo que esta configuração estrutural concorre para um menor desenvolvimento cognitivo (Noble et al., 2015).

O tema tem sido abordado em algumas revistas científicas de referência, como a Lancet, onde é referido que as alterações económicas conduzem a problemas que podem ter repercussões ao nível da saúde mental, como o desemprego, a perda de rendimentos, e o aumento do endividamento familiar (Karanikolos et al., 2013).
Por exemplo, o Ministério da Saúde da Grécia apresentou dados que apontam para um aumento do número de suicídios, na ordem dos $40 \%$, entre janeiro e maio de 2011, comparativamente com igual período do ano anterior (Economou, Madianos, Theleritis, Peppou, \& Stefanis, 2011). Já em Espanha, entre 2006 e 2010 a prevalência de problemas de saúde mental em pessoas que recorreram aos Cuidados de Saúde Primários aumentou significativamente, especialmente no que se refere a distúrbios do humor, ansiedade, somatoforme, e relacionados com o álcool; ainda assim, o maior aumento foi ao nível da prevalência da Depressão Major (Gili, Roca, Basu, McKee, \& Stuckler, 2013).

Em 2011, a World Health Organization (WHO) publicou um manual intitulado "Impact of economic crises on mental health", no qual procurou realizar uma análise da situação à data da publicação, bem como apresentar algumas sugestões relativas à manutenção dos sistemas de saúde mental durante o período de crise financeira (como o combate ao estigma, investir na área da saúde mental, continuar as reformas da saúde mental, e assegurar o universalismo nos serviços de saúde mental) (WHO, 2011). A figura 1, extraída do manual da WHO (2011) visa explicar de forma sumária a ligação entre a crise financeira e os potenciais efeios ao nível da saúde mental.

Figura 1 - Ligação entre a Crise Financeira e a Saúde Mental

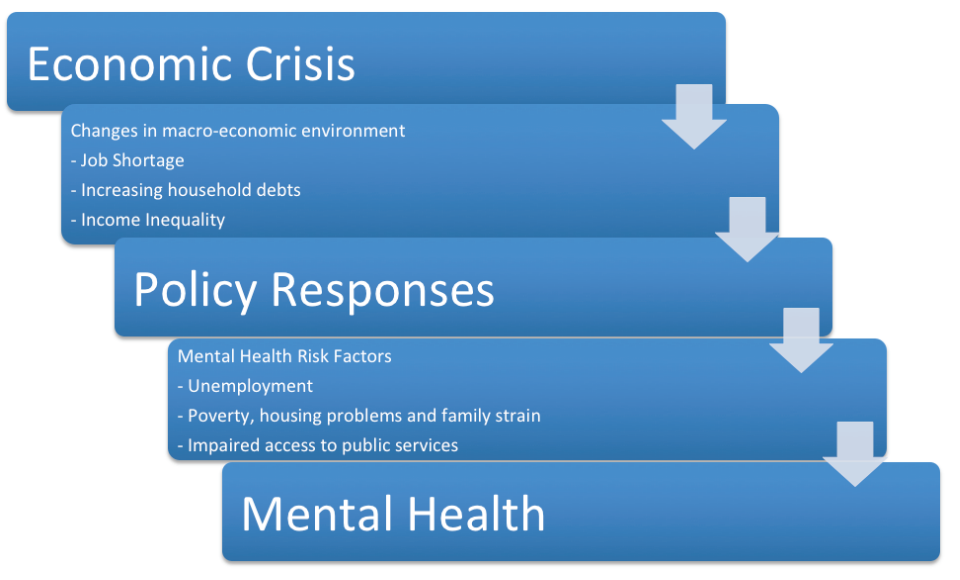

Fonte: WHO (2011) 


\section{ANÁLISE DA SITUAÇÃO ATUAL}

No que concerne ao contexto português, não se encontrou na literatura um número significativo de trabalhos centrados nos efeitos da crise financeira na saúde mental das pessoas. Porém, existem já alguns esforços nesse sentido. A título de exemplo, a Dissertação de Mestrado da autoria de Serra (2014) refere que em 2012 os portugueses se sentiam ansiosos (60\%), com humor depressivo (43\%), tristes (53\%) e com a sensação de que a vida se agravava progressivamente (95\%). Estes dados, que colocam Portugal próximo dos lugares cimeiros da Europa no que concerne aos sentimentos de ansiedade, tristeza, depressão e agravamento da vida, estão diretamente relacionados com a crise financeira e com a implementação de políticas de austeridade, na medida em que os dados revelam que quando a satisfação com a economia diminui, aumentam os sentimentos de depressão, tristeza, ansiedade e agravamento da vida, diminuindo igualmente o sentimento de calma, paz e felicidade. Adicionalmente, verificam-se relações entre a pobreza e a exclusão social, e o aumento dos sentimentos de tristeza e agravamento da vida (Serra, 2014). Ainda em Portugal, Mateus, Carvalho e Xavier (2015) realizaram uma avaliação multidimensional no sentido de atualizar o Plano Nacional de Saúde Mental 20072016, considerando os desafios que emergiram da crise financeira na Europa e subsequentes restrições orçamentais, e de definir as principais prioridades para a implementação do mesmo. Dessa análise, quatro prioridades foram definidas para a atualização do Plano Nacional de Saúde Mental 2007-2016: 1) criar um novo modelo de financiamento e gestão; 2) prosseguir o processo de desinstitucionalização; 3) avançar com a implementação dos Cuidados Continuados de Saúde Mental; 4) integrar o projeto de monitorização dos direitos humanos e promoção da qualidade dos cuidados, a ser desenvolvido em cooperação com a WHO.

De acordo com estudos epidemiológicos de Saúde Mental (Direção-Geral da Saúde [DGS], 2013; Caldas de Almeida e Xavier, 2013), Portugal tem uma das taxas mais elevadas de perturbação psiquiátrica $(22,9 \%)$, sendo as perturbações da ansiedade (16,5\%) e as perturbações depressivas $(7,9 \%)$ as mais relevantes. Verifica-se ainda um elevado consumo de psicofármacos. Tendo por referência os 12 meses anteriores ao estudo, verificavase que $23,4 \%$ das mulheres referiam ter ingerido ansiolíticos (valor mais elevado na Europa), sendo esta taxa de $9,8 \%$ nos homens.
Também se verifica que os custos associados à doença mental são muito elevados, e são ainda mais elevados quando as intervenções ao nível da promoção da saúde e da prevenção da doença mental são insuficientes. Por outro lado, os custos da doença estão associados aos custos da falta de produtividade, aos custos associados à segurança social, à diminuição de impostos, etcentre outros. A conjugação destes fatores favorece as condições para um aumento do número de pessoas com problemas e, consequentemente, com maior morbilidade mental. Por exemplo, de acordo com os dados da Organização para a Cooperação e Desenvolvimento Económico (OCDE) (2015) em cada três desempregados a receber subsídio, verifica-se que um sofre de doença mental.

É claro que sendo a "disponibilidade económica" um determinante fundamental para a Saúde Mental das pessoas, quer de forma direta (acesso ao tratamento, ...) quer de forma indireta (desemprego, expetativas, ...), a sua degradação terá sempre implicações significativas em termos de Saúde Mental. Acrescem neste contexto três fatores que parecem relevantes:

i) Tratou-se de uma crise inesperada, e por isso, não permitiu uma preparação adequada à minimização do impacto em termos de Saúde Mental;

ii) Surge aquando de um processo de desinstitucionalização em curso, com o encerramento dos hospitais psiquiátricos e a necessidade de criação de novas estruturas de natureza comunitária que possam ajudar a uma efetiva integração da pessoa com doença mental;

iii) Vive-se numa época com mudanças significativas na estrutura e dimensão das famílias, associadas na maior dos casos a uma menor disponibilidade para o suporte, onde prevalecem as famílias nucleares, tornando mais difícil o exercício do papel de prestador de cuidados.

A principal questão que se coloca é: qual o verdadeiro impacto da crise na Saúde Mental das pessoas?

i) A crise tem impacto ao nível do acesso aos cuidados de Saúde Mental, devido à diminuição do financiamento para saúde e ao nível da ausência de investimento em estruturas fundamentais para uma resposta satisfatória, essencialmente ao nível comunitário.

ii) A crise aumenta as situações de pobreza, exclusão social, redução de salários, redução da satisfação, diminuição da qualidade de vida, o que se traduz num aumento do número de suicídios, aumento da número de casos de depressão e, consequentemente, aumento do consumo de antidepressivos (Eurofound, 2014). 
iii) Impacto a nível familiar, na medida em que se transfere muitos dos cuidados para a família, mais concretamente para o prestador de cuidados. A necessidade de prestação de cuidados, associada a maiores dificuldades económicas, diminuição de apoio, por exemplo, ao nível de uma adequada preparação/capacitação, leva a que muitos familiares cuidem por amor, mas com dificuldades acrescidas, o que tem como consequência uma elevada sobrecarga e que concorre para um maior risco de aparecimento de uma perturbação mental, como a ansiedade e depressão.

iv) Impacto a nível social e económico, na medida em que interfere com a produtividade, aumenta o absentismo e as despesas com os tratamentos. Uma das consequências das dificuldades financeiras consiste em aumentar o risco de um maior número de pessoas não aderir ao tratamento, por via da incapacidade para adquirir a medicação prescrita e/ou por via da incapacidade para suportar as despesas inerentes a propostas de intervenção não farmacológicas.

\section{ESTRATÉGIAS DE MINIMIZAÇÃO DO IMPACTO DA CRISE NA SAÚDE MENTAL}

Em termos do impacto da crise na assistência em Saúde Mental salientam-se as escassas respostas comunitárias. Por outro lado, algumas respostas até existem, mas de forma esporádica. O que falta é uma articulação estruturada entre as diferentes respostas - exemplo articulação entre os departamentos de Psiquiatria, Serviços Locais de Saúde Mental (SLSM), os ACES (mais propriamente as Unidades de Saúde Familiar [USF]), as Unidades de Cuidados na Comunidade (UCC), e as Unidades de Saúde Pública (USP), pois não existindo formalmente qualquer tipo de articulação, quando a pessoa regressa a casa não tem continuidade de cuidados, sendo que em Saúde Mental esta continuidade tem um impacto determinante na eficácia das abordagens terapêuticas.

É necessário investir mais e melhor na formação dos diferentes agentes de forma a garantir a qualidade de cuidados. Falta criar padrões de qualidade das práticas de cuidados em Saúde Mental. A maioria das equipas das USF e das USP não têm profissionais qualificadas na área da Saúde Mental. Igualmente, as pessoas e as famílias das pessoas com demências necessitam de "lares", estruturas comunitárias e apoio domiciliário para esta área, com menor custo e maior qualidade de cuidados. Em termos de acesso, verificaram-se algumas melhorias no que reporta essencialmente à intervenção na doença. Existem mais hospitais com urgência psiquiátrica e consulta externa e são atendidos mais utentes do que na década de 2000, embora com menos tempo por consulta.
A principal lacuna está no acompanhamento que é efetuado às pessoas, essencialmente em termos de Enfermagem e de outras áreas.

Considera-se que respostas de base comunitária, de maior proximidade, com uma taxa de custo/benefício muito significativo poderiam minimizar o impacto da crise. A título de exemplo, destacam-se as intervenções sobre a literacia em saúde mental, psicoeducação, intervenção precoce, ou visitas domiciliárias de apoio, que poderiam ser implementadas pelos enfermeiros especialistas em Enfermagem de Saúde Mental e Psiquiátrica que exercem funções nos Cuidados de Saúde Primários, de forma a minimizarem o sofrimento das pessoas doentes e suas famílias. É fundamental formar os enfermeiros de família na área de Saúde Mental para que estes possam efetuar um rastreio e um encaminhamento de saúde mental adequado.

Importa ainda salientar, no relatório do Observatório Português dos Sistemas de Saúde (OPSS) (2015), que os cuidados de internamento são fundamentais e imprescindíveis, pois em Portugal não há respostas excessivas nesta área. Os internamentos que existem de longa duração são escassos e centram-se essencialmente nos cuidados de saúde privados (Casas de Saúde e Misericórdias). Há algumas exceções nos hospitais públicos (no Porto, Coimbra e Lisboa), mas ainda não existe qualquer resposta comunitária que possa suplementar as "camas hospitalares". Quando as Unidades de Cuidados Continuados em Saúde Mental estiverem implementadas, então alguma redução (ainda que residual) será possível, pois a maioria dos casos são "antigos", não se verificando um aumento significativo de novos casos.

Os locais para atender pessoas com problemas mentais agudos, e em situação de crise ou descompensação psíquica, necessitam de equipas alargadas e diversificadas e com experiência: expandir tudo para a comunidade é desaproveitar capacidade instalada e pode mesmo afirmar-se que parece tratar-se de uma intenção idealista. É necessário utilizar o "potencial instalado" nas Casas de Saúde, que ao longo dos tempos têm fornecido uma resposta imprescindível na área da Saúde Mental. É fundamental implementar programas de vigilância e de intervenção, essencialmente para as pessoas/grupos vulneráveis, de modo a diminuir o "peso" das variáveis que concorrem para uma maior incidência de doença mental.

É fundamental ajudar as famílias a cuidarem de si mesmas e a cuidarem dos familiares em situação de risco ou portadores de doença mental. Existe uma associação significativa entre o desemprego e a insatisfação com a vida, o que por sua vez está associado a maior ansiedade e depressão. 
Existem um conjunto de associações de promoção, de reintegração, de apoio à pessoa com doença mental que necessitam de ser avaliadas, monitorizadas de forma regular e referenciadas pela rede de referenciação em saúde mental, uma vez que em algumas situações a "doença" retira a capacidade de serem os doentes a fazêlo. É preciso garantir que não há um "aproveitamento" da vulnerabilidade da pessoa com doença mental.

\section{CONCLUSÕES}

Falta melhorar o acesso a serviços de Saúde Mental, essencialmente ao nível da promoção da saúde e prevenção da doença, ao nível do acesso às pessoas com perturbações mentais leves (ansiedade, depressão, autocontrolo, consumos de substâncias psicoativas), privilegiando as respostas não farmacológicas que possibilitam a capacitação da pessoa e não apenas o tratamento "do sinal e/ ou sintoma", com recurso a enfermeiros especialistas em Enfermagem de Saúde Mental, psicólogos, terapeutas ocupacionais, entre outros.

$\mathrm{Na}$ área infanto-juvenil o número de respostas ainda é mais insuficiente.

Faltam respostas coordenadas e articuladas nas escolas, nas universidades, que sejam promotoras da Saúde Mental, que possibilitem um diagnóstico precoce e o combate ao estigma, diminuindo o tempo que vai entre o aprecimento de um problema e a intervenção em saúde mental para muitas situações.

Falta reforçar a literacia em saúde mental, ao nível da psicoeducação e da implementação do técnico de referência para a ajuda, capacitação e avaliação das pessoas na comunidade (gestor de caso). Um outro principal problema está relacionado com a continuidade de cuidados, pois quando um doente regressa a casa, a continuidade dos cuidados que são prestados no internamento é interrompida e, por vezes, não é continuada por ninguém em termos da gestão do regime terapêutico, da capacitação para as atividades de vida diária, e/ ou da reintegração social.

Em síntese é necessário envidar esforços muito significativos, para melhorar as respostas na doença mental leve e no acompanhamento formal, organizado, estruturado e sistematizado à pessoa com doença mental após a alta clinica, de forma a evitar o número sucessivo de reinternamentos e promover uma integração plena da pessoa na comunidade. É fundamental formar e apoiar os prestadores de cuidados informais (familiares) de forma a contribuir para uma prestação de cuidados de qualidade e, por consequência, manter a pessoa dependente no seu domicílio.

\section{REFERÊNCIAS BIBLIOGRÁFICAS}

Caldas de Almeida, J., \& Xavier, M. (2013). Estudo Epidemiológico Nacional de Saúde Mental (Vol. 1). Lis $\neg$ boa. Faculdade de Ciências Médicas, da Universidade Nova de Lisboa.

Direção-Geral da Saúde (2013). Saúde Mental em Números 2014. Lisboa: Direção-Geral da Saúde.

Economou, M., Madianos, M., Theleritis, C., Peppou, L. E., \& Stefanis, C. N. (2011). Increased suicidality amid economic crisis in Greece. Lancet, 378(9801), 1459. doi: 10.1016/S0140-6736(11)61638-3.

Eurofound. (2014). Acess to Healthcare in time of crisis. Luxembourg: office of the European Union, European Foundation for the improvement of living and Working Conditions. Disponível em: http://www.eurofound.europa. eu/sites/default/files/ef_publication/field_ef_document/ ef1442en.pdf

Gili, M., Roca, M., Basu, S., McKee, M., \& Stuckler, D. (2013). The mental health risks of economic crisis in Spain: Evidence from primary care centres, 2006 and 2010. European Journal of Public Health, 23, 103-108. doi: 10.1093/eurpub/cks035

Karanikolos, M., Mladovsky, P., Cylus, J., Thomson, S., Basu, S., Stuckler, D., ...McKee, M. (2013). Financial crisis, austerity, and health in Europe. Lancet, 381, 1323-1331. doi: 10.1016/ S0140-6736(13)60102-6

Mateus, P., Carvalho, A., \& Xavier, M. (2015). The impact of the economic crisis in mental health: Redefining the priorities of mental health policies and plans. European Psychiatry, 30(Suppl. 1), 1382. doi: 10.1016/S0924-9338(15)31073-7

Noble, K. G., Houston, S. M., Brito, N. H., Bartsch, H., Kan, E., Kuperman, J. M., ...Pediatric Imaging, Neurocognition, and Genetics Study (2015). Family income, parental education and brain structure in children and adolescents. Nature Neuroscience, 18(5), 773-778. doi: 10.1038/nn.3983

OCDE. (2015). OECD High-level policy fórum on mental health and work. Session1. Adressing employment and health needs early. Issues for discussion. Hage: organization for Economic Co-operation and development (OECD). Disponível em http://www.oecd.org/mental-health-andwork-forum/documents/ISSUES-FOR-DISCUSSION.pdf

Observatório Português dos Sistemas de Saúde (2015). Acesso aos cuidados de saúde. Um direito em risco? Relatório de Primavera 2015. Acedido em http://www.opss. $\mathrm{pt} /$ node $/ 485$

Serra, F. M. A. R. V. (2014). Efeitos da crise económica na saúde mental: Portugal na União Europeia (2004-2012) (Dissertação de Mestrado). Faculdade de Economia da Universidade de Coimbra, Coimbra.

World Health Organization (2011). Impact of economic crises on mental health. Copenhaga: World Health Organization. 